\title{
Reacción de antígenos de Leishmania (Leishmania) mexicana con sueros de pacientes con leishmaniosis cutánea de Sinaloa, México
}

\author{
Patricia Guadalupe Salazar-Mejía, QFB, ${ }^{(1)}$ Celia Rosa Tejeda-Aguirre, M en C,(2) \\ Héctor Samuel López-Moreno, D en C.(I)
}

Salazar-Mejía PG,Tejeda-Aguirre CR, López-Moreno HS. Reacción de antígenos de Leishmania (Leishmania) mexicana con sueros de pacientes con leishmaniosis cutánea de Sinaloa, México. Salud Publica Mex 2010;52:165-169.

\section{Resumen}

Objetivo. Detectar los antígenos de Leishmania (Leishmania) mexicana que reaccionan con sueros de pacientes con leishmaniosis cutánea (LC) de Sinaloa, México. Material y métodos. Un extracto crudo de $L$. (L.) mexicana fue usado como antígeno para Western blots 2-D empleando sueros de cinco pacientes con LC y controles originarios de Sinaloa, México, durante el 2008. Resultados. Cinco antígenos fueron detectados sólo por los sueros de los cinco pacientes estudiados; estos son: $26 \mathrm{kDa}$ (pl 7.8), $27 \mathrm{kDa}$ (pl 8.I), $28 \mathrm{kDa}$ (pl 8.6), $29 \mathrm{kDa}$ (pl 8.5) y $31 \mathrm{kDa}$ (pl 9.0). Conclusiones. Se detectaron nuevos antígenos de $L$. (L.) mexicana potencialmente inmunodominantes, lo que sugiere a este parásito como el agente causal de la LC en Sinaloa.

Palabras clave: Leishmania mexicana; antígenos; leishmaniosis cutánea
Salazar-Mejía PG,Tejeda-Aguirre CR, López-Moreno HS.

Reaction of Leishmania (Leishmania) mexicana

antigens by sera of patients with cutaneous

leishmaniasis from Sinaloa, Mexico.

Salud Publica Mex 2010;52:165-169.

\begin{abstract}
Objective. To detect Leishmania mexicana antigens reacting with sera of patients with cutaneous leishmaniasis $(\mathrm{CL})$. Material and methods. A crude extract of $L$. mexicana was used as antigen for 2-DWestern blot using sera from 5 patients with $\mathrm{CL}$ and controls from Sinaloa, Mexico during 2008. Results. Five antigens were detected in the five infected patients analyzed; their molecular weights and isoelectric points were: $26 \mathrm{kDa}$ (pl 7.8), $27 \mathrm{kDa}$ (pl 8.I), $28 \mathrm{kDa}$ (pl 8.6), $29 \mathrm{kDa}(\mathrm{pl} 8.5$ ) and $31 \mathrm{kDa}$ (pl 9.0). Conclusion. New potentially immunodominant L. mexicana antigens were detected, suggesting that this parasite could be the species responsible for human infection in Sinaloa.
\end{abstract}

Key words: Leishmania mexicana; antigens; cutaneous leishmaniasis

(I) Laboratorio de Biomedicina, Facultad de Ciencias Químico Biológicas de la Universidad Autónoma de Sinaloa. Sinaloa, México.

(2) Departamento de Vectores y Zoonosis SSA. Sinaloa, México.

Fecha de recibido: 6 de febrero de 2009 - Fecha de aceptado: 12 de noviembre de 2009 Solicitud de sobretiros: Dr. Héctor Samuel López-Moreno. Laboratorio de Biomedicina, Facultad de Ciencias Químico Biológicas, Universidad Autónoma de Sinaloa. Av. Américas y Blvd. Universitarios S/N. 80000, Culiacán, Sinaloa. Correo electrónico: hsamlo@uas.uasnet.mx 
$\mathrm{L}^{2}$ a leishmaniosis es una parasitosis causada por diferentes especies de protozoarios intracelulares obligados pertenecientes al género Leishmania, transmitida al ser humano cuando una hembra hematófaga de dípteros del género Lutzomyia (en América) o Phlebotomus (en Europa, Asia y África), ${ }^{1-3}$ regurgita promastigotes después de alimentarse.

La leishmaniosis puede clasificarse en tres formas principales: la primera es la leishmaniosis cutánea (LC, que puede ser localizada o difusa), la segunda es la leishmaniosis destructiva mucocutánea (LMC) y la tercera, que puede ser fatal, conocida como leishmaniosis visceral (LV o Kala-Azar). ${ }^{1}$ En México, la LV es endémica en Chiapas, Guerrero y Morelos; la LMC en Tabasco y Chiapas y la LC es endémica en Campeche, Chiapas, Coahuila, Jalisco, Michoacán, Nayarit, Nuevo León, Oaxaca, Tamaulipas, Quintana Roo, Tabasco, Veracruz, Yucatán y recientemente Sinaloa. ${ }^{3}$ La LC fue descrita por primera vez en Sinaloa en 1994 y desde entonces a la fecha se han reportado más de 100 casos; $^{4}$ sin embargo, se carece de estudios que aborden la identificación de antígenos del parásito.

Se considera a Leishmania (Leishmania) mexicana como la especie que causa la LC en México. ${ }^{3}$ El diagnóstico definitivo de la LC está basado en datos clínicos y epidemiológicos asociados con una técnica diagnóstica de laboratorio. Las técnicas diagnósticas más comúnmente utilizadas para la identificación de esta parasitosis son la reacción cutánea de Montenegro y el examen histopatológico, ${ }^{5}$ sin embargo, estas técnicas carecen de sensibilidad. Actualmente el estándar de oro para la identificación de especies de Leishmania es la electroforesis multilocus de enzimas (MLEE). ${ }^{6}$ Una de las desventajas de esta técnica es que requiere del cultivo y aislamiento del parásito, un procedimiento que es susceptible de contaminación bacteriana y tarda semanas o meses para su conclusión, por lo que esta técnica no es útil para la toma de decisiones terapéuticas. Por otro lado, los métodos serológicos son sensibles y específicos, como el ELISA (Ensayo por inmunoabsorción ligado a enzimas), permiten el procesamiento de un mayor número de muestras y los resultados se obtienen en horas. La sensibilidad y especificidad del método de ELISA depende de la calidad del antígeno utilizado, por lo que el objetivo de este trabajo fue la detección de antígenos inmunodominantes de $L$. (L.) mexicana que son reconocidos por sueros de pacientes con LC originarios del estado de Sinaloa, los cuales puedan ser evaluados posteriormente en el diseño y validación de un ELISA para el diagnóstico de la LC, o bien ayuden al diseño de protocolos de inmunización para contribuir al desarrollo de una vacuna contra esta parasitosis.

\section{Material y métodos}

\section{Sueros}

La población en estudio comprendió a individuos con cuadro clínico sugestivo de LC que acudieran al Centro de Salud de Mazatlán, Sinaloa, de enero a diciembre del año 2008, y como controles negativos a voluntarios sanos de la misma área, con características similares en cuanto a edad y sexo. En todos los sujetos se obtuvo el consentimiento informado de acuerdo con las normas establecidas por el Comité de Ética de la Secretaría de Salud de Sinaloa. En el caso de los individuos con cuadro clínico sugestivo de LC el criterio de inclusión fue el establecimiento del diagnóstico de certeza mediante la demostración del parásito por impronta. Se obtuvieron los sueros de los pacientes con diagnóstico de LC, así como de los controles, los cuales fueron analizados.

\section{Extracto crudo de L. (L.) mexicana (ECLm)}

El extracto crudo de L. (L.) mexicana (ECLm) fue obtenido por sonicación a partir de "pellets" de cultivo de promastigotes de la cepa MHOM/MX/92/UAY68 de L. (L.) mexicana, gentilmente donada por la Dra. Patricia Talamás Rohana, los cuales fueron resuspendidos en $80 \%$ de amortiguador de rehidratación (BRH) para electroforesis de doble dimensión (2-D), el cual contiene 8M UREA, 2\% CHAPS, 50mM DTT, 0.2\% de anfolitas Bio-lyte y trazas de azul de bromofenol (Bio-Rad), y $20 \%$ de un cóctel de inhibidores de proteasas (Sigma). El ECLm fue clarificado por centrifugación. Las proteínas del ECLm fueron precipitadas y lavadas tres veces con ocho volúmenes de acetona (JT Baker), resuspendidas en BRH y posteriormente cuantificadas por el método de Bradford (Sigma).

\section{Electroforesis de doble dimensión (2-D)}

Las muestras fueron sometidas a isolelectroenfoque empleando tiras de gel con gradiente de $\mathrm{pH}$ inmovilizado (IPGs, Bio-Rad). Posteriormente fueron sometidas a electroforesis de doble dimensión (2-D) en geles de poliacrilamida al $12.5 \%$ de acuerdo a protocolos previamente establecidos. Estos geles fueron teñidos con azul de Coomassie G-250 (Bio-Rad), y desteñidos para el conteo de las proteínas del ECLm. Alternativamente, duplicados de estos geles fueron transferidos a membranas de nitrocelulosa de $0.22 \mu \mathrm{m}$ (Hybond-ECL, Amersham) y empleadas como base para los Western blots. 
Western blot

Después de bloquear las membranas con leche descremada al 5\%, éstas fueron incubadas con los sueros de los pacientes con LC parasitológicamente confirmada, diluidos 1:100, o con los sueros de los testigos a la misma dilución. Posteriormente, las membranas fueron incubadas con anticuerpos anti-IgG humana conjugada a peroxidasa (Zymed) y finalmente, la reacción fue revelada con diaminobencidina (Research Organics) y $\mathrm{H}_{2} \mathrm{O}_{2}$ (JT Baker).

\section{Resultados y discusión}

La leishmaniosis cutánea (LC) en el estado de Sinaloa, México es considerada como una parasitosis emergente con un registro histórico oficial de 100 casos, la mayoría de los cuales se han presentado en la zona sur de Sinaloa. ${ }^{4}$ Actualmente el diagnóstico se realiza mediante exámenes histopatológicos, los cuales poseen baja sensibilidad, apenas alcanzan 50\%, y están limitados para el procesamiento de un gran número de muestras, además de que requieren disponer de técnicos de laboratorio con gran pericia para la observación de los amastigotes. Una alternativa diagnóstica la ofrecen los métodos inmunológicos como el ELISA, que poseen una mayor sensibilidad y excelente especificidad; sin embargo, dependen de las características inmunoquímicas del antígeno. Para contribuir al diseño de un método de diagnóstico inmunológico y eventualmente de una vacuna, se realizó el presente estudio encaminado a la detección y posterior identificación de los antígenos inmunodominantes del protozoario parásito Leishmania (L.) mexicana, considerado el principal agente etiológico de la LC en México. ${ }^{3}$ Para ello se obtuvo un extracto crudo de L. (L.) mexicana (ECLm) a partir de cultivos de promastigotes, para posteriormente realizar una electroforesis de doble dimensión que evidenció mediante tinción con azul brillante de Coomassie G-250 un total de 137 proteínas contabilizadas mediante el software PDQuest 7.3.1 (Bio-Rad) (dato no mostrado). Réplicas de esos geles fueron utilizadas para su transferencia a membranas de nitrocelulosa que sirvieron de soporte sólido para la realización de Western blot individuales mediante los sueros de cinco pacientes con LC localizada originarios del estado de Sinaloa, México, confirmados parasitológicamente (figura 1), y el mismo número de sueros de testigos no parasitados. Los cinco pacientes incluidos en este estudio presentaron lesiones ulcerosas en el pabellón auricular (figura 1A) y al realizarles las improntas fue posible observar varios macrófagos infectados con amastigotes de Leishmania sp., (figura 1B).

Al revelar los Western blots fue posible detectar que varios antígenos reaccionan con los sueros de los pacientes con LC, pero en especial identificamos cinco proteínas antigénicas potencialmente dominantes, ya que son reconocidas en los cinco pacientes. Se determinaron los pesos moleculares y puntos isoeléctricos de cada uno de ellos: $26 \mathrm{kDa}$ (pI 7.8), $27 \mathrm{kDa}(\mathrm{pI} 8.1), 28$ $\mathrm{kDa}(\mathrm{pI} 8.6), 29 \mathrm{kDa}$ (pI 8.5), y 31kDa (pI 9) (figura 2).
A)

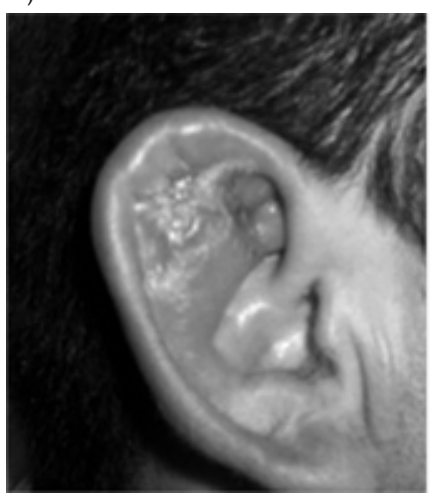

B)

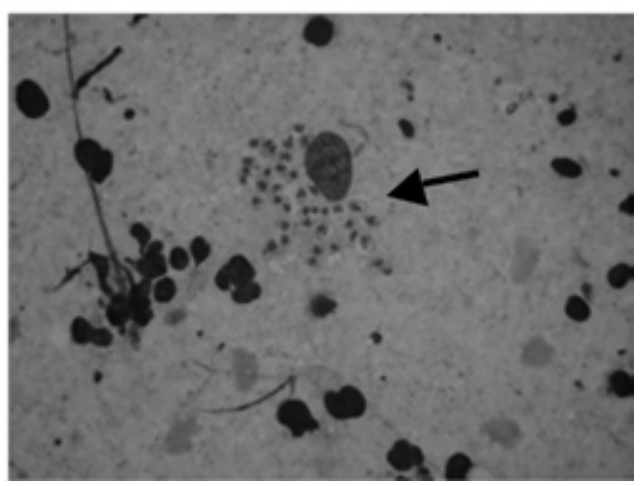

Figura I. Paciente con leishmaniosis cutánea. A) se muestra la imagen de un paciente del estado de Sinaloa, Mé-

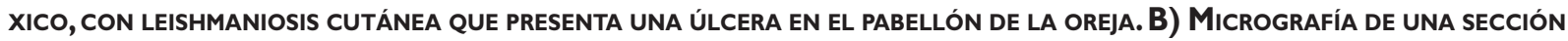
DE LA IMPRONTA OBTENIDA DEL PACIENTE EN A, DONDE SE OBSERVA UN MACRÓFAGO MULTIPARASITADO CON AMASTIGOTES DE LEISHMANIA SP. (FLECHA) 


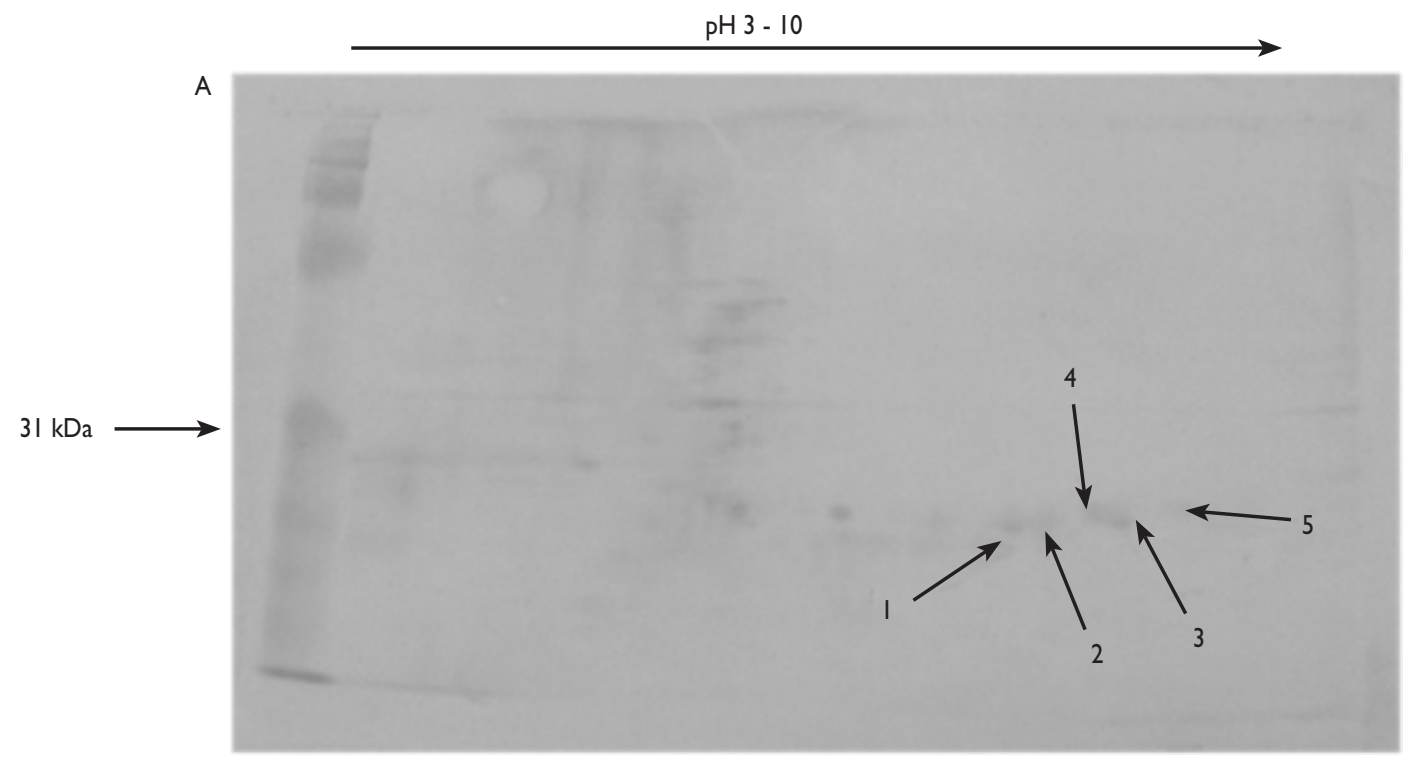

Figura 2. Detección de antígenos de Leishmania (Leishmania) mexicana por sueros de pacientes Con LC del estado de Sinaloa, México. A) Western blot 2-D de uno de los cinco pacientes con LC donde se observa el GRUPo de cinco

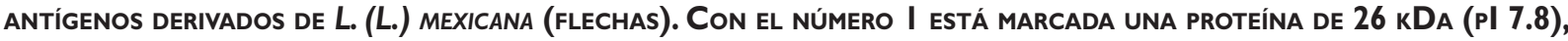
CON EL NÚMERO 2, UNA de 27 KDA (PI 8. I), LA NÚMERO 3 de 28 kDA (PI 8.6), LA 4 de 29 kDA (PI 8.5), Y FINALMENTE LA 5 de 3 I KDA (PI 9). CADA SUERO FUe ANALIZADO POR DUPLICADO, Y TODOS LOS PACIENTES RECONOCIERON A LOS 5 ANTíGENOS

Existen diferentes reportes donde se han detectado e identificado diferentes antígenos derivados de diferentes especies de Leishmania, ;,8 sin embargo, ninguno coincide completamente con las características inmunoquímicas de los reportados en este trabajo. Osland y colaboradores ${ }^{8}$ reportaron antígenos prominentes de $L$. (L.) aethiopica detectados mediante Western blot de una dimensión, pero su peso molecular fue de $25 \mathrm{kDa}$, inferior a los detectados en este trabajo. Kamoun-Essghaier y colaboradores ${ }^{10}$ reportan un grupo de seis antígenos derivados de promastigotes de L. (L.) infantum evidenciados mediante Western blot de 2-D con pesos moleculares cercanos a los detectados en este trabajo, en el rango de 30-36 kDa, pero con diferente pI. ${ }^{10}$ Esto sugiere que los antígenos derivados de $L$. (L.) mexicana reportados en el presente estudio son novedosos. Sin embargo, es necesario continuar con los estudios a fin de incluir un mayor número de pacientes, así como secuenciar los diferentes antígenos para identificar su naturaleza bioquímica y diseñar un ELISA con alguno de estos antígenos o con combinaciones de los mismos. Adicionalmente, sería de gran relevancia evaluar la participación de estos antígenos en la relación hospedero-parásito, lo cual permitiría incrementar nuestro conocimiento para el diseño de una vacuna o bien para comprender los mecanismos inmunológicos protectores o permisivos en la leishmaniosis, que continúa siendo un importante problema de salud en México y en otros países del mundo.

\section{Agradecimientos}

Los autores agradecen a la Dra. Patricia Talamás Rohana por el donativo del cultivo de L. mexicana. También agradecen al CECYT Sinaloa y al PROFAPI 2008/105 por el financiamiento otorgado para la realización de este proyecto. Finalmente, agradecen la asesoría técnica de la $\mathrm{M}$ en C. Karen Pineda Hidalgo.

\section{Referencias}

I. Comité de Expertos de la OMS. Las leishmaniasis. Ginebra: Organización Mundial de Salud, 1984:793.

2. Salotra P, Singh R. Challenges in the diagnosis of post kala-azar dermal leishmaniasis. Indian J Med Res 2005; 123:295-3 I0.

3. Hernández-Ruiz J, Becker I. Linfocitos T citotóxicos CD8 ${ }^{+}$en la leishmaniasis cutánea. Salud Publica Mex 2006;48:430-439.

4. Córdova-Uscanga C,Albertos-Alpuche NE,Andrade-Naváez FJ,

Canto-Lara SB. Leishmaniasis: estudio epidemiológico preliminar en una 
localidad de la zona endémica del estado de Tabasco. Salud Publica Mex 1993;35:345-350.

5. Canto-Lara SB, Cárdenas-Marrufo MF,Vargas-González A,AndradeNarváez F]. Isoenzyme characterization of Leishmania isolated from human cases with localized cutaneous leishmaniasis from state of Campeche, Yucatan Peninsula, Mexico.Am J Trop Med Hyg 1998;58:444-447. 6. Peres-Ferreira M, Ferreira AM, Passeri MM, Machado J, De Castro JF. Sensitivity of an immunoenzymatic test for the detection of anti-L. braziliensis antibodies compared to other tests used for the diagnosis of American cutaneous leishmaniasis. Rev Inst Med Trop S Paulo 2006;48(4):215-217.

7. Rioux JA, Lanotte G, Serres E, Pratlong F, Bastein P, Perieres J.Taxonomy of Leishmania. Use of isoenzymes. Suggestions for a new classification.Ann Parasitol Hum Comp 1990; 65: I I - 25.
8. Osland A, Beyene D, Ashenafi S, Beetsma A. Isolation and characterization of recombinant antigens from Leishmania aethiopica that react with human antibodies. Infect Immunity 1992;60(4): I 368-I374.

9. Forgber M, Basu R, Roychoudhury K, Theinert S, Roy S, Sundar S, et al. Mapping the antigenicity of the parasites in Leishmania donovani infection by proteome serology. Plos One 2006; I (I):I-I I,e40.

I0. Kamoun-Essghaier S, Guizani I, Strub JM,Van Dorsselaer A, Mabrouk $\mathrm{K}$, Ouelhazi L, et al. Proteomic approach for characterization of immunodominant membrane-associated 30- to 36-kilodalton fraction antigens of Leishmania infantum promastigotes, reacting with sera from mediterranean visceral leishmaniasis patients. Clin Diag Lab Immunol 2005; I2(2):310-320. 